\title{
Coronary Flow Interruption Following Balloon Rupture in an Elderly Patient
}

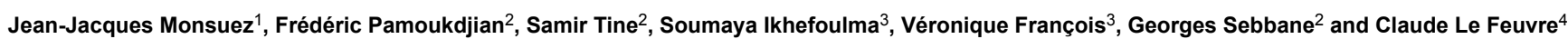 \\ ${ }^{1}$ Department of Cardiology, University Hospital, Paris Seine Saint Denis, Hospital René Muret, France \\ ${ }^{2}$ Department of Geriatry, Avicenne Hospital, University Hospitals of Paris Seine Saint Denis, France \\ ${ }^{3}$ Department of Geriatry, Hospital René Muret, University Hospitals of Paris Seine Saint Denis, France \\ ${ }^{4}$ Department of Cardiology, Pitié-Salpêtrière Hospital, Heart Institute, University of Paris 6, Faculty of Medicine, France
}

"Corresponding author: Jean-Jacques Monsuez, AP-HP, Hôpital René Muret, Policlinic Medical Avenue du Docteur Schaeffner, F-93270 Sevran, France, Tel: 33-1-41525884; Fax: 33-1-141525863; E-mail: jean-jacques.monsuez @ aphp.fr

Rec Date: Jun 27, 2016; Acc Date: Sep 26, 2016; Pub Date: Sep 28, 2016

Copyright: ( $) 2016$ Monsuez JJ, et al. This is an open-access article distributed under the terms of the Creative Commons Attribution License, which permits unrestricted use, distribution, and reproduction in any medium, provided the original author and source are credited.

\section{Introduction}

As a result of the increased life expectancy, elderly with acute coronary syndromes (ACS) referred to coronary catheterization facilities for emergency percutaneous coronary interventions (PCI) increase in numbers. Whereas PCI has proven effective in this setting, management of elderly with ACS remains a challenging issue. Concerns about benefit-risk ratio include physiological age and associated co-morbidities such as renal and/or cognitive impairment. More specific conditions should also be taken into account when deciding an invasive approach, including presentation with symptoms of heart failure (HF) and the extent of coronary artery lesions. This case report relates of an unusual but potentially harmful complication of percutaneous revascularization that may be observed in elderly patients with severely calcified coronary lesions.

\section{Case Report}

An 88-year-old woman was admitted to hospital for increasing shortness of breath lasting for 2 days. Her past history included prior surgery for hip fracture and for gall bladder lithiasis migration-related angiocholitis 5 and 10 years before, respectively. She also had mild to moderate chronic obstructive lung disease associated with past cigarette smoking and bronchial hyper reactivity.

There was no cough, wheezing, nor chest pain. Blood pressure was $140 / 90 \mathrm{mmHg}$, the pulse was 100 per minute, regular, and breath rate was 22 per minute. Physical examination revealed normal heart sounds. The lung bases were clear at auscultation. Palpation of the abdomen was normal. The electrocardiogram showed no abnormalities. There was no pulmonary edema on a chest X-ray, which also showed a slightly enlarged cardiac silhouette.

Routine laboratory examinations showed mild leukocytosis (WBC count: $11,900 / \mathrm{mm}^{3}, 90 \% \mathrm{PMN}$ ), no anemia (hemoglobin: $12.1 \mathrm{~g} / 100$ $\mathrm{mL}$ ), plasma creatinine level $78 \mu \mathrm{mol} / \mathrm{L}$. Plasma troponin I level was normal $(0.05 \mu \mathrm{g} / \mathrm{L})$.

As plasma Elisa D-dimers levels were slightly increased, a duplex Doppler ultrasound examination of the lower extremities was performed, but excluded deep venous thrombosis.

A blood gas sampling showed $\mathrm{pH} 7.36, \mathrm{PaCO}_{2} 49.5 \mathrm{mmHg}$, and $\mathrm{PaO}_{2} 62 \mathrm{mmHg}$. As the pro-BNP levels were increased $(2,822 \mu \mathrm{g} / \mathrm{mL})$, a treatment with diuretics and oxygen was started in the emergency department. The patient substantially improved $\left(\mathrm{PaO}_{2}: 80 \mathrm{mmHg}\right)$ and was subsequently discharged to the department of medicine.
Two days later, she complained of recurrent increasing dyspnea, without chest pain, wheezing nor crepitations over the lung bases. The electrocardiogram showed sinus rhythm with a heart rate 116 per minute and recent onset electrocardiographic changes suggesting STelevation anterior myocardial infarction. Blood pressure was 90/65 $\mathrm{mmHg}$. Troponin I was $1.01 \mu \mathrm{g} / \mathrm{L}$. A 2-dimensional echocardiography (2-DE) showed impaired septal and apical wall motion, with a reduced left ventricular ejection fraction (LVEF: $40 \%$ ).

Emergency coronary angiography was performed after a therapy with aspirin $250 \mathrm{mg}$, clopidogrel $300 \mathrm{mg}$ and low molecular weight heparin was administered. A significant stenosis (80\%) was seen on segment 2 of the left anterior descending coronary artery (LAD). There was no coronary thrombus within the lumen of the vessel, which was, however, heavily calcified. No other critical stenosis was found. The left main coronary artery and circumflex coronary artery were plaque- and stenosis-free. A $40 \%$ stenosis was seen in segment 1 of the right coronary artery. Primary coronary angioplasty was attempted using a 2 $\mathrm{mm}$ balloon inflated within the lumen of the mid LAD stenosis. As repeat inflations failed to achieve any effective dilatation, a $2.5 \mathrm{~mm}$ balloon was placed with stepwise increases in inflation pressure. Balloon rupture occurred during a 22 atm inflation and instantaneously resulted in a prolonged no flow within the target vessel lasting for 30 seconds (Figure 1), followed by a slow flow associated with severe hypotension (systolic blood pressure $70 \mathrm{mmHg}$ ) which lasted for a few minutes. This slow flow was ultimately reverted after intracoronary administration of adenosine ( 3 bolus of $36 \mu \mathrm{g}$ each). At the end of the procedure, a TIMI-3 flow grade was obtained, with a residual stenosis accounting for $40 \%$ of the target vessel lumen. Blood pressure was $110 / 60 \mathrm{mmHg}$.

Troponin levels rose to $3.79 \mu \mathrm{g} / \mathrm{L} 24$ hours later and subsequently returned to normal values. A week later, the patient said her shortness of breath had disappeared. The electrocardiogram showed an improved repolarization pattern in leads V1 to V4, without constitution of any $\mathrm{Q}$ wave. Septal motion abnormalities substantially improved, with a LVEF ranging between $50 \%$ and $55 \%$.

\section{Discussion}

Elderly patients with ACS often present with atypical or absent chest pain, complaining more frequently of dyspnea or manifestations of worsening heart failure [1,2]. Delay in seeking medical care, misleading clinical symptoms and electrocardiographic changes, as well as a lower rate of patients referred for early invasive strategy affect the prognosis of ACS in the elderly [2,3], whereas several studies have 
shown that PCI is as safe and effective in this population [3-5]. Efficacy of PCI may however be limited by severe target vessel calcifications, which are a common finding in the elderly [5].

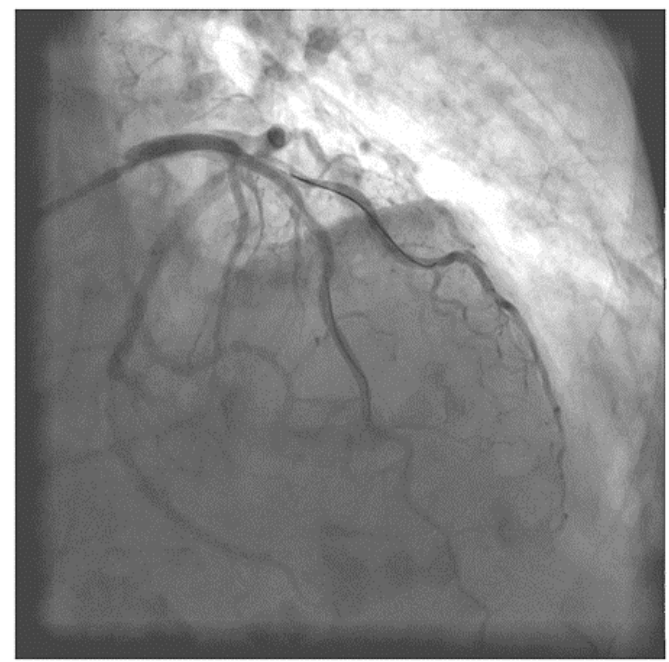

Figure 1: No flow/slow flow within the LAD, associated with normal flow within the remaining coronary arteries.

The patient reported herein combines an initial presentation with HF symptoms and severely calcified coronary lesions. Whereas the former has been recognized as a leading indication for primary PCI, the latter, conversely, is associated with an increased risk of PCI.

Balloon rupture (and sometimes multiple balloon rupture) during additional high-pressure inflation has been reported to be elicited by calcified lesions protruding into the coronary lumen [6-9]. In the study by Chan et al. balloon rupture occurred in 110 of 2984 patients who underwent PCI. Although an event-free post-PCI course was observed in 102 of them (93\%), 8 patients (7\%) experienced severe adverse procedural injuries, including 3 deaths, 1 cardiogenic shock, 3 ACS, 1 tamponade. Emergency surgery was performed in 4 of these 8 cases [10]. Balloon rupture may also result in coronary artery dissection $[11,12]$ which may also be associated with coronary rupture [13-15], balloon entrapment [16], balloon fragments embolization [17] or air embolism [18].

Balloon rupture occurs mainly during high pressure inflations. In the study by Dübel et al. the shape of the balloon became distorded with increasing pressure, and a linear relationship was observed between balloon diameter and increasing pressure. Most balloons increased by more than $15 \%$ in diameter over the nominated diameter before rupture occurred [19].

Although well known by most interventional cardiologists, the phenomenon of no flow/slow flow following balloon rupture remains poorly understood. Distal embolism of microparticles and/or fragmented thrombus in patients treated for ACS, air embolism or vascular spasm involving large vessels in the setting of elective PCI have been advocated. Microcirculatory vasoconstriction has also been advocated, resulting from either an abrupt drop in coronary pressure or a jet lesion into the coronary lumen after the contrast medium embedded within the balloon is propelled into the coronary flow $[20,21]$.
The present study shows a potentially harmful complication of balloon angioplasty elicited by high inflation pressures and severely calcified coronary lesions. Transient hemodynamic failure may adversely affect elderly patients, especially those with HF or LV diastolic dysfunction associated with myocardial ischemia. In our patient, the no flow/slow flow phenomenon resulted in a sustained drop in blood pressure that reverted only after several intracoronary administration of adenosine. This case study would be worthy of further research to assess the frequency and the consequences of this complication in a large sample of patients in whom stenting is not performed, especially when platelet inhibition is contraindicated.

Accordingly, decision making for primary PCI in elderly patients with ACS should take into account the extent of target vessel calcifications in association with the patient's baseline clinical condition. Facing with severely calcified coronary lesions, the risk of balloon rupture and PCI failure should be considered in a pre-inflation benefit-risk ratio analysis.

\section{References}

1. Brieger D, Eagle KA, Goodman SG, Steg PG, Budaj A, et al. (2004) Acute coronary syndromes without chest pain, an underdiagnosed and undertreated high-risk group: insights from the Global Registry of Acute Coronary Events. Chest 126: 461-469.

2. Mehta RH, Rathore SS, Radford MJ, Wang Y, Krumholz HM (2001) Acute myocardial infarction in the elderly: differences by age. J Am Coll Cardiol 3: 736-741.

3. Bauer T, Koeth O, Jünger C, Heer T, Wienbergen H, et al. (2007) Effects of an invasive strategy on in-hospital outcome in elderly patients with nonST elevation myocardial infarction. Eur Heart J 28: 2873-2878.

4. Kaehler J, Meinhertz T, Hamm C (2006) Coronary interventions in the elderly. Heart 92: 1167-1171.

5. Chauhan M, Kuntz R, Kalon K, Cohen D, Popma J, et al. (2001) Coronary stenting in the aged. J Am Coll Cardiol 37: 856-862.

6. Zellner C, Sweeney JP, Ko E, Sudhir K, Chou TM (1997) Use of intravascular ultrasound in evaluating repeated balloon rupture during coronary stenting. Cathet Cardiovasc Diagn 40: 52-54.

7. Kahn JK, Hartzler GO (1990) Balloon rupture due to lesion morphology during coronary angioplasty. Cathet Cardiovasc Diagn 21: 89-91.

8. Angelini P (1990) Balloon catheter coronary angioplasty: balloon rupture. Cathet Cardiovasc Diagn 20: 150-151.

9. Mascarenhas DA, Saperia GM, Benotti JR (1993) Balloon rupture complication. Cathet Cardiovasc Diagn 28: 266-268.

10. Chan AW, Rabinowitz A, Webb JG, Dodek A, Jarochowski M, et al. (1999) Adverse events associated with balloon rupture during percutaneous coronary intervention. Can J Cardiol 15: 962-966.

11. Kwan T, Huber MS, Jani H, Feit A (1994) Multiple balloon rupture during coronary angioplasty: a case report. Angiology 45: 891-896.

12. Gilutz H, Weinstein JM, Ilia R (2000) Repeated balloon rupture during coronary stenting due to a calcified lesion: an intravascular ultrasound study. Catheter Cardiovasc Interv 50: 212-214.

13. Rothschild R, Voda J (1990) Coronary artery dissection caused by angioplasty balloon rupture. Cathet Cardiovasc Diagn 19: 26-29.

14. Christiansen EH (2007) Unusual balloon rupture during direct stenting with a Taxus Express stent in a venous graft complicated by vessel rupture: a device-related fatal event. J Interv Cardiol 20: 23-25.

15. LeMay MR, Beanlands DS (1990) Pinhole balloon rupture during coronary angioplasty causing rupture of the coronary artery. Cathet Cardiovasc Diagn 19:91-92.

16. Colombo A, Skinner JM (1990) Balloon entrapment in a coronary artery: potential serious complications of balloon rupture. Cathet Cardiovasc Diagn 19: 23-25. 
Citation: Monsuez JJ, Pamoukdjian F, Tine S, Ikhefoulma S, François V, et al. (2016) Coronary Flow Interruption Following Balloon Rupture in an Elderly Patient. J Gerontol Geriatr Res 5: 344. doi:10.4172/2167-7182.1000344

Page 3 of 3

17. Schräder R, Krause E, Blum U, Kober G (1990) Rupture and fragmentation of a polyethylene terephtalate balloon catheter in a calcified coronary artery stenosis. Z Kardiol 79: 199-202.

18. Kahn JK, Hartzler GO (1990) The spectrum of symptomatic air embolism during balloon angioplasty: causes, consequences and management. Am Heart J 119: 1374-1377.
19. Dübel HP, Gliech V, Bunk G, Kubat B, Schneider M, et al. (1997) Rupture of PTCA balloons: an in vitro study. Z Kardiol 86: 968-974.

20. Kahn JK (1993) Slow coronary flow complicating elective balloon angiography in postthrombolytic patients. Coron Artery Dis 4: 61-65.

21. Kraft M (1999) Dependence on PTCA-pressure rupture of the balloon used under certain conditions. Biomed Tech (Berl) 44: 182-184. 